\title{
STATISTICS OF TIMES OF MINIMUM LIGHT OF 1,140 ECLIPSING BINARY STARS
}

\author{
C.-H. Kim \\ Department of Astronomy and Space Science \\ Chungbuk National University, Cheongju, Korea \\ kimch@ast.chungbuk.ac.kr
}

\section{J. M. Kreiner}

Mt. Suhora Observatory

Pedagogical University, Krakow, Poland

sfkreine@cyf-kr.edu.pl

\section{I.-S. Nha}

The Nha Il-Seong Museum of Astronomy

Yechon-gun, Kyongbuk, Korea

slisnha@chollian.net

\begin{abstract}
In this paper the collected times of minimum light of 1,140 eclipsing binary stars were statistically analyzed.

Keywords: Times of minimum light, eclipsing binary stars, statistics

\section{Introduction}

The study of period changes of eclipsing binary stars provides important and direct clues in understanding certain astrophysically interesting topics, such as: the internal structures of the stars, tests of the theory of general relativity, the three-body problem, mass transfer from one component to the other, mass loss from one (or both) component(s), and magnetic activities of the stars. This study has been usually based on the relatively simple $(O-C)$ diagram, formed from observed times of minimum light $(O)$ minus those calculated from an ephemeris $(C)$, plotted against time or the number of orbital revolutions.
\end{abstract}


All timings for numerous eclipsing binary stars have been systematically collected into a database. At the moment, the database contains about 142,000 timings for 4,092 eclipsing binaries and is as complete as possible through mid - 2002. Assorted statistics for the timings of all stars is listed in Table 1. An atlas of $(C)$ diagrams for 1,140 eclipsing binary stars has been published by Kreiner, Kim \& Nha (2001). The detailed information on the Atlas can be found at a Web address (http://www.as.wsp.krakow.pl/o-c/). With some exceptions, most of the stars in the Atlas fulfill three criteria: (1) at least 20 minima have been timed; (2) these minima span at least 2,500 cycles; and (3) the 2,500 cycles are distributed over at least 40 years.

Table 1. Statistics of collected times of minimum light.

\begin{tabular}{cc|ccccc|cr}
\hline \multicolumn{2}{c|}{ Type } & \multicolumn{5}{c|}{ Method } & \multicolumn{2}{c}{ Year } \\
\hline Pri & Sec & Vi & P & Pg & E & CCD & Start & End \\
\hline 119,690 & 22,417 & 64,581 & 43,098 & 11,623 & 18,303 & 4,502 & 1783 & 2002 \\
\hline
\end{tabular}

These 1,140 stars correspond to about $28 \%$ of the objects in the database and are represented by 91,798 timings, i.e., about $65 \%$ of the entries in the database. In this paper some statistics of times of minimum light in the Atlas are presented.

\section{Statistics of Times of Minimum light}

The number of the observed minima in a given year is shown in Fig. 1a and $1 \mathrm{~b}$. One can see a decrease in the number of minima during and immediately after the First and Second World Wars. The first use of photographic methods occurred at the beginning of the 20th century, with the greatest activity in the 1930s. One can see the increasing use

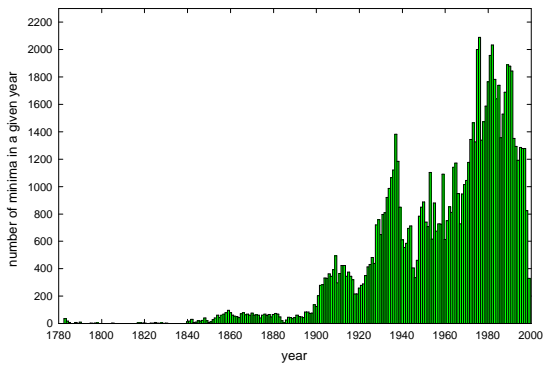

Figure 1a. Total number of minima in a given year.

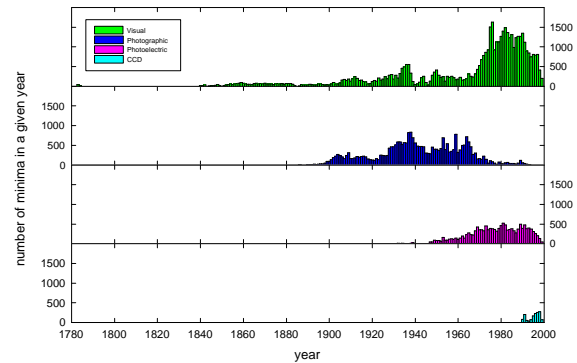

Figure $1 b$. Number of minima according to methods of observation. 
of photoelectric methods from 1948, with a corresponding decline in photography. Similarly, the use of photoelectric photometry has declined since 1995 with the increase in use of CCDs. CCD minima have been published since about 1990 . The rise in numbers of visual minima between 1970 and 2000 reflects the increased activity of amateur observers.

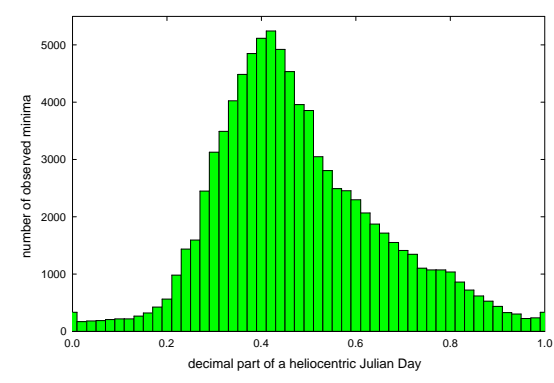

Figure 2. Number of minima relative to the decimal part of a timing.

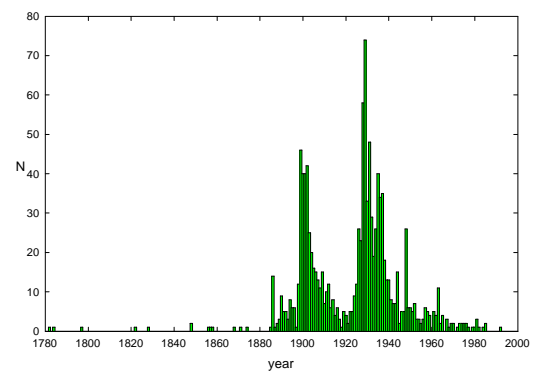

Figure 3. Number of eclipsing binaries discovered in a given year.

The number of minima relative to the decimal part of a timing is shown in Fig. 2. One can see that most of the minima occur at 0.4 Julian day, i.e. close to $22^{\mathrm{h}} 00^{\mathrm{m}}$ UT. This shows that most minima were observed from Europe. There is also a small plateau around 0.8 which reflects the contribution of American observers.

In Fig. 3 there plotted the number of eclipsing binaries discovered in a given year. A discovery is considered to be the first published minimum. The two peaks at about 1900 and 1929 are due to photographic observations. Discoveries made by the Hipparcos satellite have not been included.

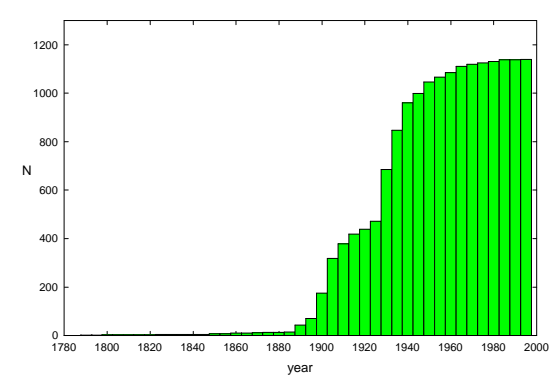

Figure 4. Number of stars with minima recorded before a given year.

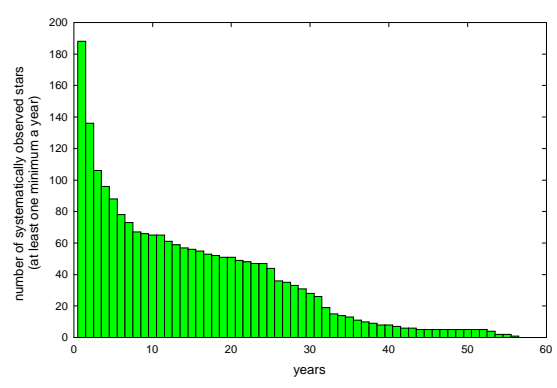

Figure 5. Number of systematically observed stars. 
Number of stars with minima recorded before a given year is shown in Fig. 4 where the number of stars for which minima have been observed rises sharply after 1900 .

Statistics for the number of stars observed systematically in the years before 2000 is shown in Fig. 5. "Observed systematically" means that at least one minimum was observed each year.

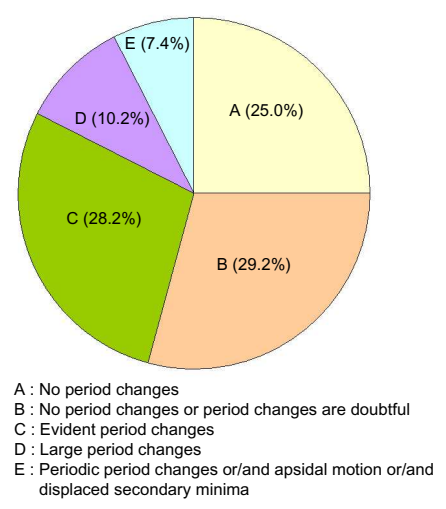

Figure 6. A percentage of period changes of stars included in the Atlas.

Finally, a percentage for period changes of 1,140 stars in the Atlas is shown in Fig. 6. One can see that about $54 \%$ of the stars have no signs of period changes while $46 \%$ show definite changes of period.

\section{Acknowledgments}

One of us (C.-H. K) acknowledges the financial support of Korea Research Foundation (KRF-2002-015-CP0150).

\section{References}

Kreiner, J. M., Kim, C.-H., and Nha, I.-S. (2001), An Atlas of O-C Diagrams of Eclipsing Binary Stars, Parts 1-6, Cracow: Pedagogical University Press. 